\title{
Respiratory symptoms of megaesophagus
}

\author{
Fabio Di Stefano, ${ }^{1}$ Lorenzo Migliorato ${ }^{2}$ \\ ${ }^{1}$ Pneumology, Allergy and Respiratory Physiopathology Unit; ${ }^{2}$ Radiology Unit, Spirito Santo Hospital, Pescara, Italy
}

\begin{abstract}
Megaesophagus as the end result of achalasia is the consequence of disordered peristalsis and the slow decompensation of the esophageal muscular layer. The main symptoms of achalasia are dysphagia, regurgitation, chest pain and weight loss, but respiratory symptoms, such as coughing, particularly when patients lie in a horizontal position, may also be common due to microaspiration. A 70-year old woman suffered from a nocturnal cough and shortness of breath with stridor. She reported difficulty in swallowing food over the past ten years, but had adapted by eating a semi-liquid diet. Chest X-ray showed right hemithorax patchy opacities projecting from the posterior mediastinum. Chest computed tomography scan showed a marked dilatation of the esophagus with abundant food residues. Endoscopy confirmed the diagnosis of megaesophagus due to esophageal achalasia, excluding other causes of obstruction, such as secondary esophagitis, polyps, leiomyoma or leiomyosarcoma. In the elderly population, swallowing difficulties due to esophageal achalasia are often underestimated and less troublesome than the respiratory symptoms that are caused by microaspiration. The diagnosis of esophageal achalasia, although uncommon, should be considered in patients with nocturnal chronic coughs and shortness of breath with stridor when concomitant swallowing difficulties are present.
\end{abstract}

\section{Introduction}

Megaesophagus is commonly a late consequence of esophageal achalasia ${ }^{1}$ which is a motility disorder involving both the smooth muscle layer and lower esophageal sphincter (LES). It is characterized by incomplete LES relaxation, increased LES tone and the lack of peristalsis of the esophagus. ${ }^{2}$ This disorder has no known underlying cause, and only a very small proportion of cases occur secondary to Chagas disease (an infectious disease common in South America). ${ }^{3}$ Megaesophagus can also result from esophageal obstruction due to cancer or fibrosis, ${ }^{4}$ or to a tight gastric band that is used to treat obesity. ${ }^{5}$

The main symptoms of achalasia are dysphagia,

Correspondence: Fabio Di Stefano and Lorenzo Migliorato, Spirito Santo Hospital, Pescara, Italy.

E-mail: f.distefano@aliceposta.it - Imigliorato@tiscalinet.it

Key words: esophageal achalasia, megaesophagus, microaspiration, respiratory symptoms.

Conflict of interests: the authors declare no potential conflict of interests.

This work is licensed under a Creative Commons Attribution NonCommercial 3.0 License (CC BY-NC 3.0).

CCopyright F. Di Stefano and L. Migliorato, 2013

Licensee PAGEPress, Italy

Italian Journal of Medicine 2013; 7:53-55

doi:10.4081/itjm.2013.53 the regurgitation of undigested food, weight loss and chest pain. The chest pain that is experienced may be mistaken for a heart attack because it can be extremely painful. Dysphagia tends to become progressively worse over time and to involve both fluids and solids. Some people may also experience coughing, stridor, wheezing and other respiratory symptoms, particularly when lying in a horizontal position, because food and liquid, including saliva, are retained in the esophagus and may be inhaled into the lungs with microaspiration. ${ }^{6}$ Aspiration can be more serious and cause pneumonia or airway obstruction due to inhaled materials.

Treatment typically involves pneumatic dilatation or surgery, such as Heller myotomy. ${ }^{7}$ Following pneumatic dilatation or surgery, proton pump inhibitors can help to prevent gastroesophageal reflux. A partial fundoplication or wrap is generally added during surgery to prevent excessive reflux.

We report a case of megaesophagus due to esophageal achalasia with swallowing difficulties. This is often underestimated and less troublesome than the respiratory symptoms that are caused by microaspiration.

\section{Case Report}

Over the course of several months, a 70-year old woman suffered from a nocturnal cough and shortness of breath with stridor. She had no previous history of respiratory or cardiac disease. The family physician had scheduled her for a cardiological examination with an electrocardiogram and echocardiogram; parameters were within the norm. She also had an ear, nose and 
throat examination with fiberoptic laryngoscopy that showed mild laryngeal hyperemia. Recent routine blood tests, such as a complete blood cell count, erythrocyte sedimentation rate, and renal and liver function tests, were all normal. No thyroid masses were detectable, and a chest auscultation revealed decreased breath sounds in the right hemithorax.

A careful assessment of her medical history revealed that the patient had reported difficulty in swallowing food over the past ten years. In the past few months, this dysphagia had worsened, but she had adapted by eating very slowly and ingesting a semiliquid diet. Furthermore, she reported frequent coughing, not only during the night and in a supine position but also particularly in the left decubitus position.

Chest X-ray showed right hemithorax patchy opacities projecting in the posterior mediastinum (Figure 1). Chest computed tomography scan revealed a severely dilated and tortuous esophagus with abundant retained food, such as that which occurs in achalasia (Figure 2A-C).

A nasogastric tube was inserted to aspirate the contents of the esophagus and the patient was then referred for an endoscopy. This excluded other causes of obstruction, such as cancer or fibrosis, confirming

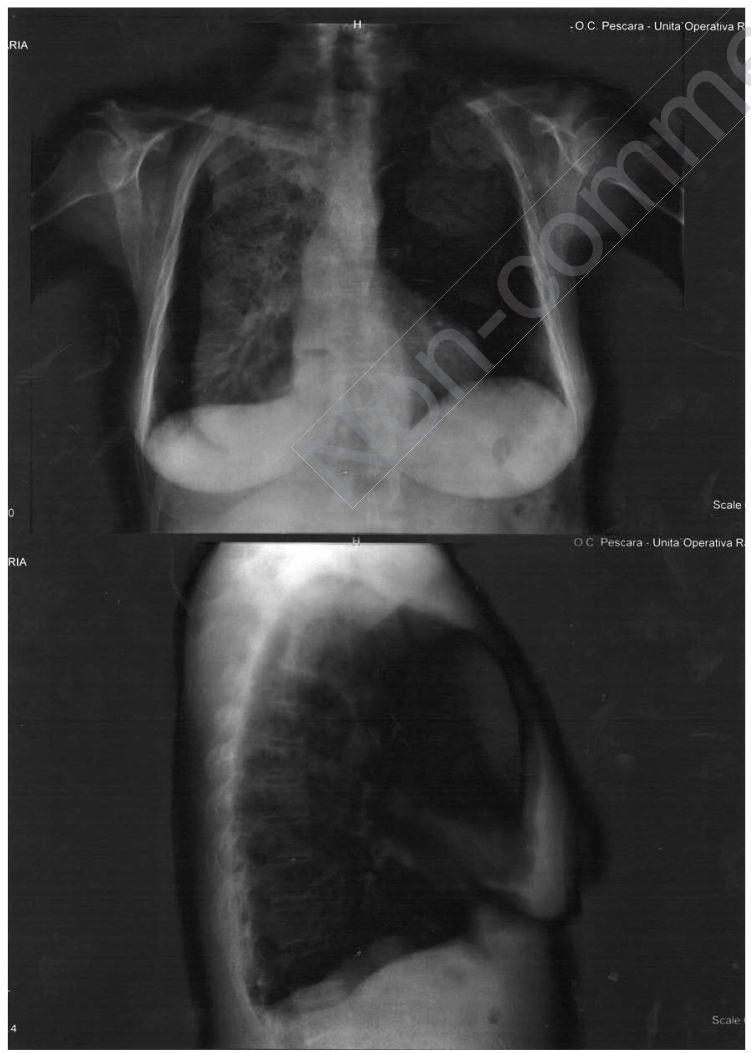

Figure 1. Chest X-ray (antero-posterior and latero-lateral projection) showing right hemithorax patchy opacities projecting in the posterior mediastinum. the diagnosis of esophageal achalasia. The lower esophageal sphincter was tight but was successfully dilated using a balloon. Nevertheless, considering the severity of the condition, the patient was also referred for further surgical treatment of the achalasia, but she declined any surgical intervention at that time.

(A)

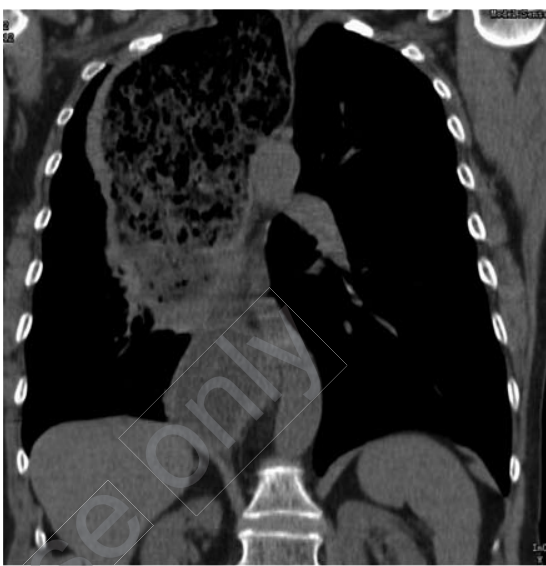

(B)

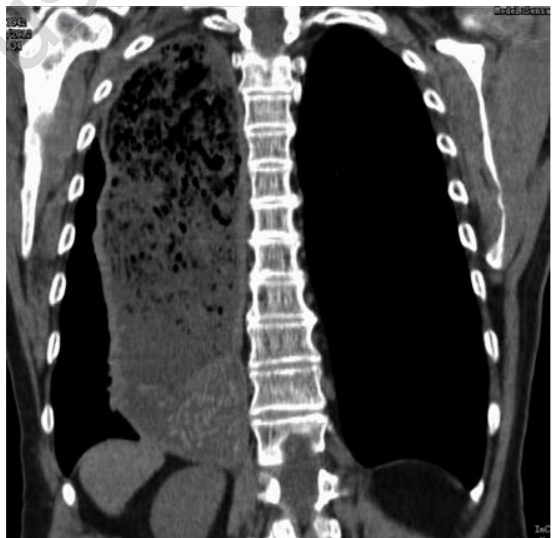

(C)

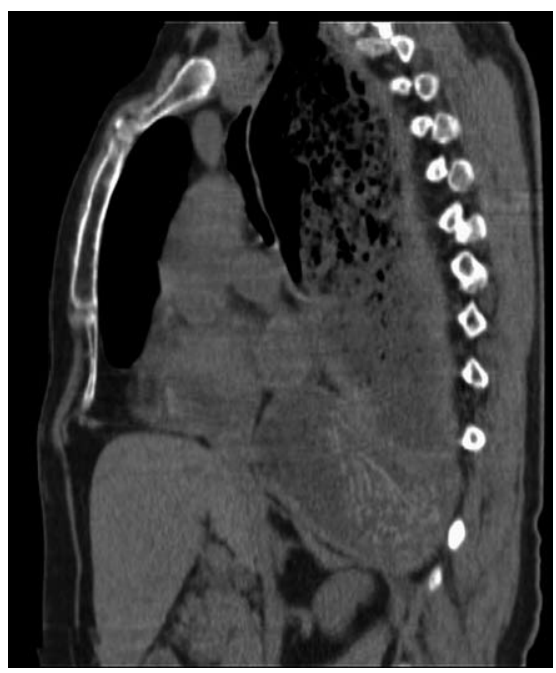

Figure 2. Chest computed tomography scan (A-B frontal sections; $C$ sagittal section) revealing megaesophagus with abundant retained food residues, such as that which occurs in achalasia. 
The patient gave informed consent to treatment and to the publication of the results.

\section{Discussion and Conclusions}

In esophageal achalasia, the slow decompensation of the muscular layer causes severe dilatation in the long term, which is a characteristic of megaesophagus.

Diagnosis depends primarily on a detailed clinical history that may lead to the administration of more specific investigations, such as a barium swallow, esophageal manometry and endoscopy, which is generally performed to rule out the possibility of cancer.

In an emergency setting, the diagnosis may be suspected following a simple chest X-ray showing patchy opacities projecting from the posterior mediastinum. This is a radiological sign of striking dilatation of the esophagus containing alimentary material. Diagnosis is straightforward with chest computed tomography. This allows disease severity to be evaluated for possible emergency treatment and helps in the planning of future therapeutic management.

Although uncommon, the diagnosis of esophageal achalasia should be considered in elderly patients with respiratory symptoms, which typically include a cough and shortness of breath with stridor in either the nocturnal or supine position, when concomitant swallowing difficulties are present. In the elderly population, swallowing difficulties are often underestimated and less troublesome than the respiratory symptoms that are caused by microaspiration.
Any delay in the diagnosis of this exceptional condition in the elderly is critical because serious consequences, such as aspiration pneumonia and airway obstruction due to inhaled alimentary material, may occur. Some authors have indeed reported sudden deaths secondary to megaesophagus that were attributed not only to food asphyxia but also to the exacerbation of pre-existing underlying disease. ${ }^{8}$

\section{References}

1. Lewandowski A. Diagnostic criteria and surgical procedure for megaesophagus - a personal experience. Dis Esophagus 2009;22:305-9.

2. Park W, Vaezi M. Etiology and pathogenesis of achalasia: the current understanding. Am J Gastroenterol 2005;100:1404-14.

3. Dantas RO, Aprile LR. Esophageal contractions in Chagas' disease and in idiopathic achalasia. J Clin Gastroenterol 2005;39:863-8.

4. Spechler SJ, Castell DO. Classification of oesophageal motility disorders. Gut 2001;49:145-51.

5. Arias IE, Radulescu M, Stiegeler R, et al. Diagnosis and treatment of megaesophagus after adjustable gastric banding for morbid obesity. Surg Obes Relat Dis 2009;5:156-9.

6. Sinan H, Tatum RP, Soares RV, et al. Prevalence of respiratory symptoms in patients with achalasia. Dis Esophagus 2011;24:224-8.

7. Richter JE, Boeckxstaens GE. Management of achalasia: surgery or pneumatic dilation. Gut 2011;60:869-76.

8. Schalinski S, Guddat SS, Tsokos M, Byard RW. Megaesophagus and possible mechanisms of sudden death. J Forensic Sci 2009;54:216-9. 